\section{Understanding sexual practices, attitudes and sexual health service provision in the over 50 s}

\author{
Dr Sandra Vracar, Dr Manjula Pammi, Dr Ruth Taylor
}

\section{Introduction}

- Evidence shows a rise in the prevalence of STIs in the over 50 population - "the middle-aged spread"1

- In the 45-64 year olds, 1 in 10 people are using barrier contraception ${ }^{2}$

- Prevalence of Chlamydia in women over 50 has risen by $95 \%{ }^{3}$

- Similar trends are also observed for Gonorrhoea ${ }^{4}$

- Possible explanations for the above are: the baby-boomer effect (particularly the population born between 1950-1960, new partners/high divorce rates, lack of awareness of STIs and poor sexual health service provision for this cohort

\section{Aims}

We performed a service evaluation to examine attitudes and sexual practices of the over 50 s in order to:

- Attempt to identify common trends and patterns in risk taking behavior in this group

- Recognize gaps in service provision and identify any ways to improve this

\section{Method}

- Anonymous qualitative questionnaires were created and piloted to a group of 10 people attending our busy city centre GUM clinic

- These questionnaires were then analysed and re-formatted accordingly, and opportunistically distributed to 50 clinic attendees between the months of September-November 2015

- The questionnaires also contained an information sheet explaining the study, as well as a consent form

\section{Patient Demographics}

The female cohort compromised of only Caucasian straight-identifying women $70 \%$ men identified as straight, 26\% gay, 87\% Caucasian, $9 \%$ Afro-Caribbean, $4 \%$ Pakistani

\section{Results}

- A greater diversity in patient demographics was found within the male cohort than the female

- 21 out of 27 women and 16 out of 23 men self-identified as "not single" with the majority being unmarried. Most women reported having 6-10 partners in their lifetime, whilst most men reported $10+$ partners

- Interestingly, when asked about current relationship status, 11 women and 7 men said they were practicing celibacy

- Of women who were sexually active, 14 were between ages 50-60, and one over 70. 14 sexually active men were aged 50-60 and two were over 70

- When answering questions about attitudes towards one-night stands, sex out of wedlock and same-sex relationships, the great majority expressed liberal opinions.

- 50\% of both men and women currently took between 1-5 medications and reported their general health as "good"

- Most people appeared to be happy with attending services as they are currently delivered, but a small number (4 women and 2 men) felt that there could be improvements

\section{How often do you attend for STI screening?}

Women

Men
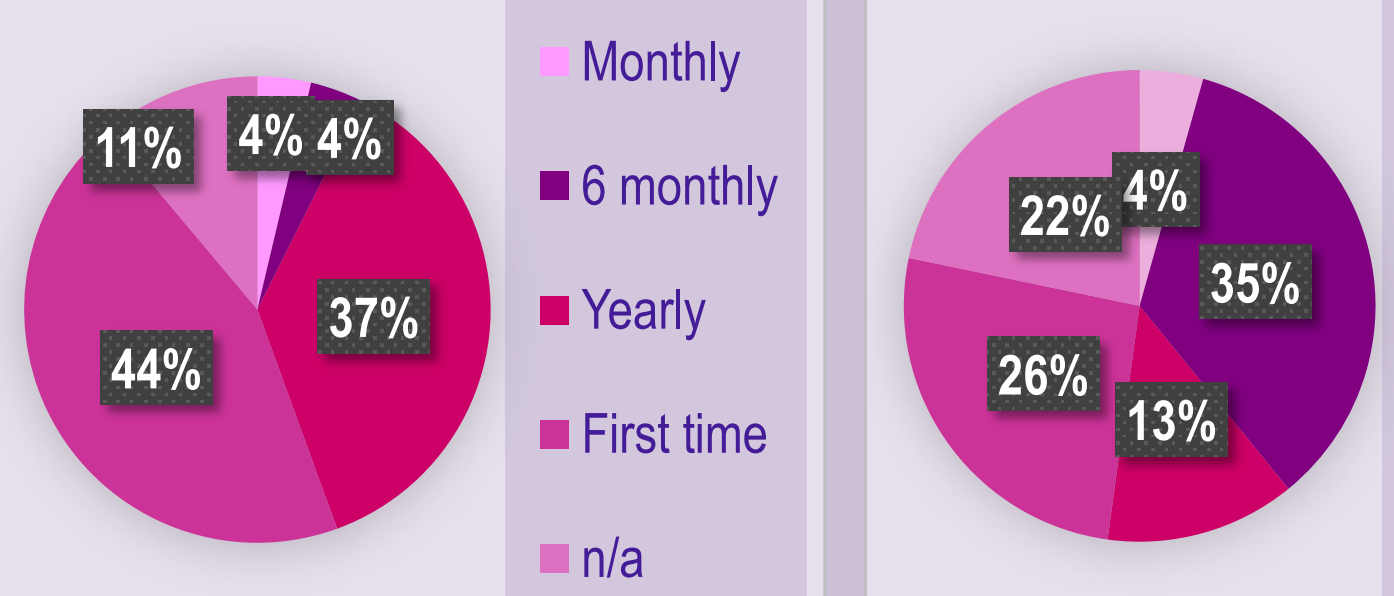

Monthly

- 6 monthly

- Yearly

- First time

n/a

\section{References}

(1) Family Planning Association: People Over 50 Relationships and Sexual Health, 2010

(2, 3, 4) GOV.UK statistics; Sexually Transmitted Infections and Chlamydia Screening, England: 2015

\section{SEXUAL HEALTH}

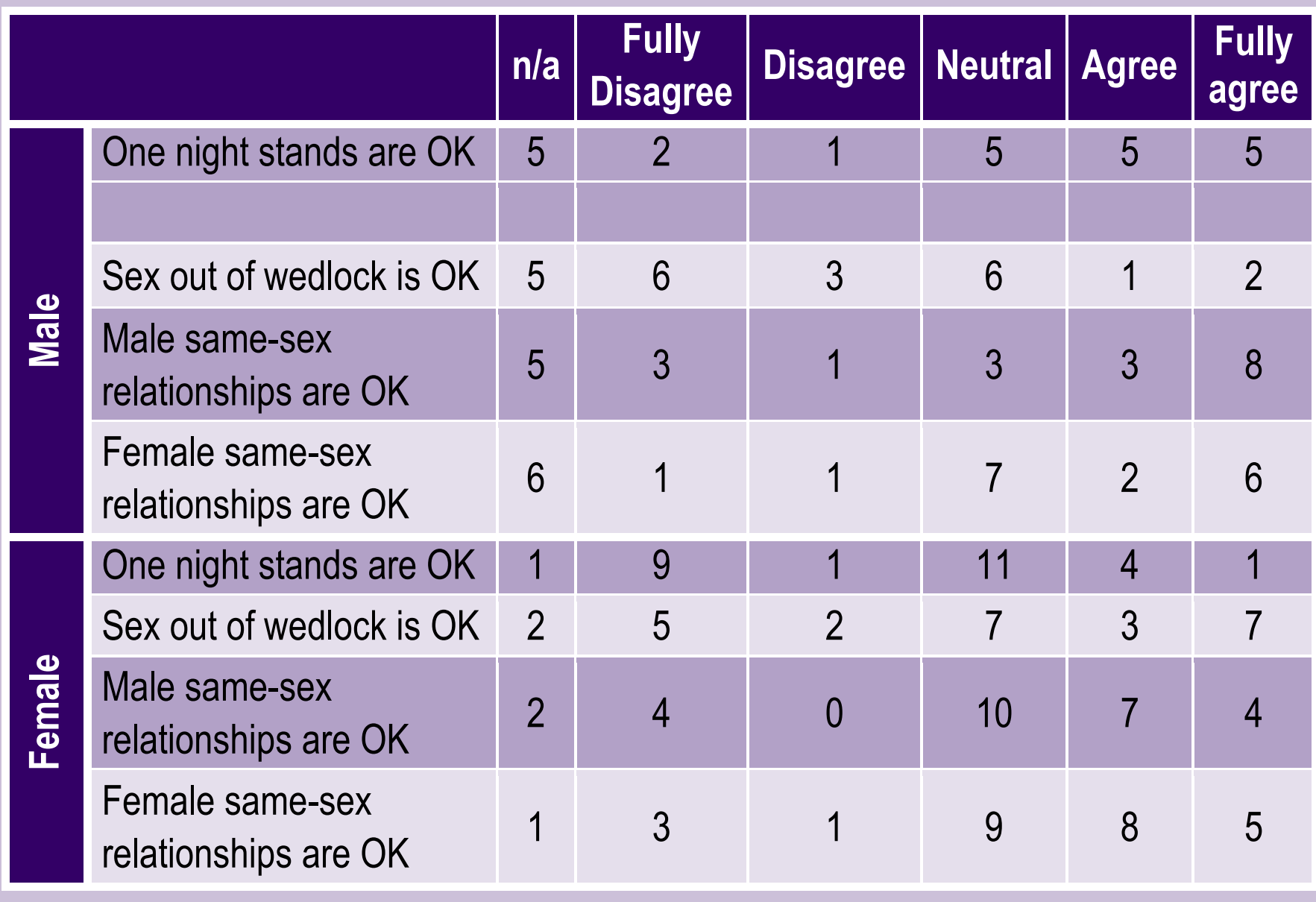

How often do you use barrier contraception? (condoms)

Women

Men

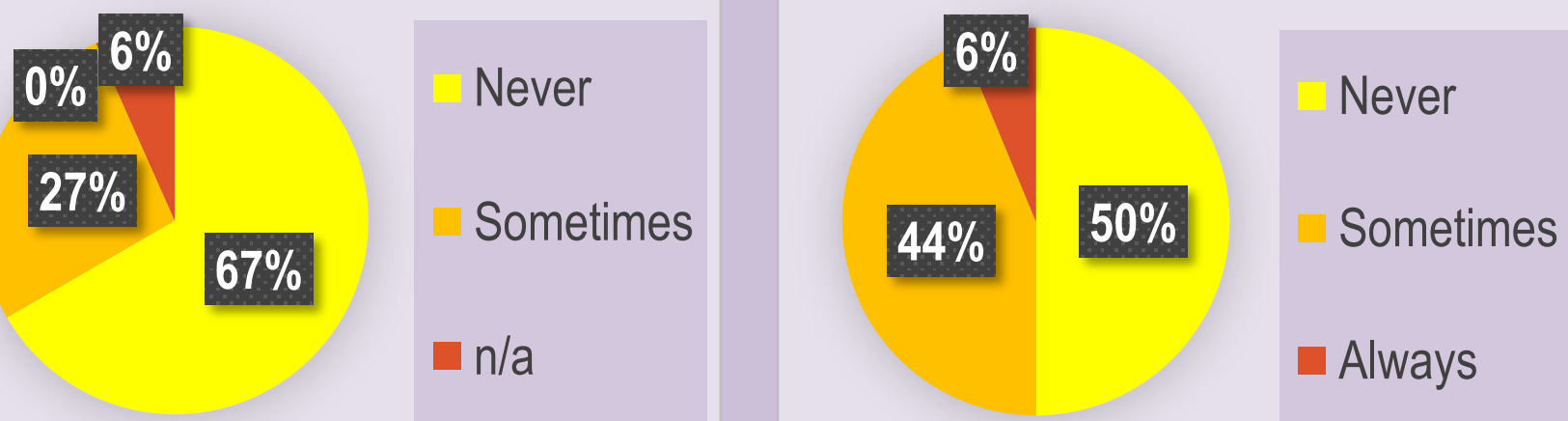

Have you heard of these 5 STIs? (chlamydia, gonorrhoea, HIV, syphilis, herpes simplex)

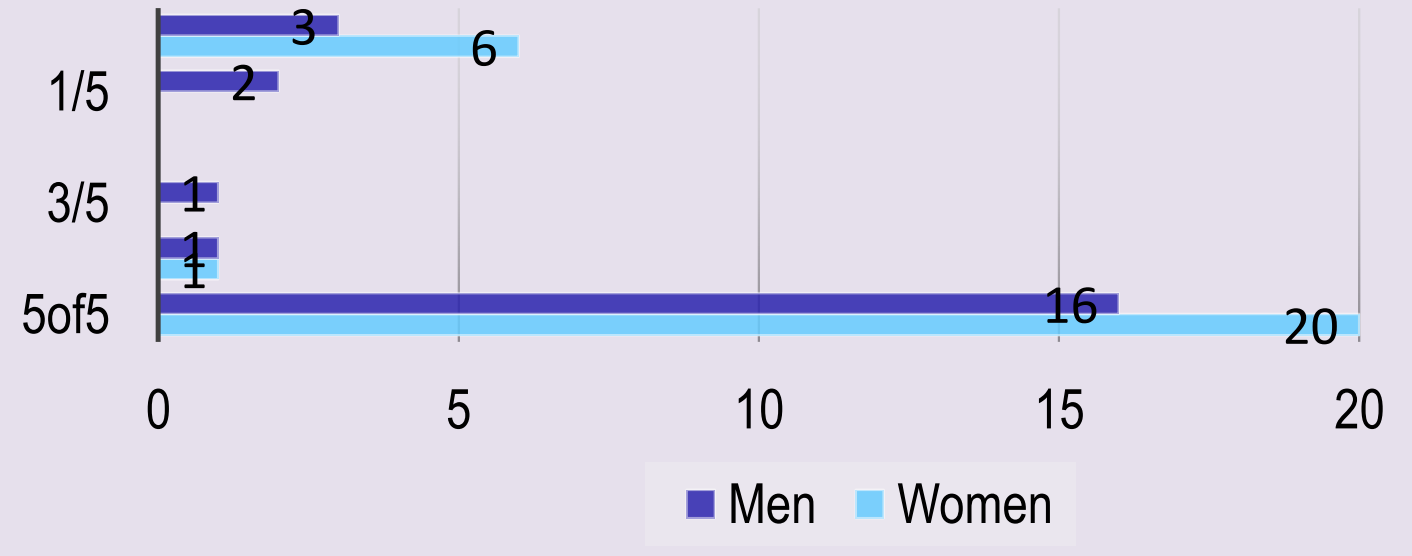

\section{Discussion}

- Current hypotheses for the recent rise in STIs in the over 50 s hold true for the cohort in this study: our participants exhibited high rates of divorce, variable engagement with regular STI testing and frequent disuse of condoms

- Reasons for not using condoms were: "no pregnancy risk/hysterectomy/menopause", "marriage/long-term relationship", "don't like it"

- Most participants showed good awareness for the common STIs (chlamydia, gonorrhoea, HIV, syphilis and herpes simplex)

- All our female participants were white Caucasian heterosexual. This may suggest an access issue for ethnic minorities or women who have sex with women. Sample size may also be an issue

- Most of the cohort held liberal views regarding extra-marital sex, same-sex relationships and casual sex, despite stereotypes about the older generation

- Some participants in the study have made suggestions to improve access to services. These include better advertising campaigns to help raise awareness and tackle stigma towards sexual health of older people

- $28 \%$ admitted being too embarrassed to talk to their GPs about sexual health

- Polypharmacy was common, with over 50\% of men and women using between 1-5 prescribed medications at any one time. This makes safe prescribing challenging

\section{Conclusion}

This survey showed that sexual risk taking is not confined to the younger age groups. Older adults do access mainstream sexual health services, but there are suggestions from our work that barriers around stigma and embarrassment do still exist, particularly for ethnically diverse women.

\section{Thank You}

Special thanks to Nottingham City Hospital Genitourinary Medicine Clinic staff for continued help practically and academically in realising this project. Finally, thank you to the patients, for their continued feedback and care in filling in the questionnaires and agreeing to take part in the study. 